\title{
From Influenza-Induced Acute Lung Injury to Multiorgan Failure
}

\author{
B. M. Tang, T. Cootes, and A. S. McLean
}

\subsection{Introduction}

Influenza viruses are among the most common causes of human respiratory infection, causing high morbidity and mortality. In the United States, influenza results in approximately 200,000 hospitalizations and 36,000 deaths during a seasonal epidemic [1]. During a pandemic, up to $50 \%$ of the population can be infected by influenza, increasing the number of deaths [2]. In 1918, the worst influenza pandemic recorded in history caused up to 50 million deaths worldwide, with elderly, infants, and people with underlying illness having the highest risk of fatality [3].

The modes of death in severe influenza patients are well recognized. They range from hypoxic respiratory failure to multiorgan failure. However, it remains unclear which host factors drive these processes. A better understanding of the host factors associated with severe disease is clinically important; it may help discover pathogenic pathways that determine disease progression and enable researchers to identify new therapeutic targets. Identifying new therapeutic targets is particularly important given that currently available antiviral therapy (e.g., neuraminidase inhibitors) has limited efficacy in reducing influenza-related fatality. Therefore,

\footnotetext{
B. M. Tang $(\bowtie)$

Department of Intensive Care Medicine, Nepean Hospital, Kingswood, NSW, Australia

Centre for Immunology and Allergy Research, Westmead Institute for Medical Research, Westmead, NSW, Australia

e-mail: benjamin.tang@sydney.edu.au

T. Cootes

Department of Infectious Diseases and Immunology, Central Clinical School, University of Sydney, Darlington, NSW, Australia
}

\author{
A. S. McLean \\ Department of Intensive Care Medicine, Nepean Hospital, Kingswood, NSW, Australia
}


delineating influenza-related host factors is a critical first step in improving patient outcome in influenza infection. Here, we examine the host factors underpinning both innate and adaptive immune response to influenza virus (Box 35.1).

\section{Box 35.1: Innate and Adaptive Immune Responses in Influenza Infection}

Innate immune response

Alveolar macrophages-resident local immune cells in lung; early contact with influenza virus; initiate phagocytosis and antigen presentation of influenza virus to activate other immune cells. Neutrophils - first wave of recruited immune cells to lung; critical in antiviral response; also aid activation of CD8 T cells. However, can exacerbate tissue inflammation in infected lung.

Monocytes - influx elicits potent inflammatory cascade; also aid the activation of CD8 T cells.

Natural killer cells - kill influenzainfected host cells; important not only for innate immune response but also for initiating adaptive immune response. Dendritic cells - cross-present influenza virus antigens or peptides to CD4 and CD8 T cells; also potent producer of antiviral molecules such as interferons.
Adaptive immune response

B cells - produce influenza virus-specific antibodies in a CD4 T-cell-dependent manner; important in humoral response to influenza virus infection; important for vaccine development.

Cytotoxic CD8 T cells-major driver of the cellular response; enable killing of influenza virus and elimination of virally infected host cells; critical for viral clearance and resolution of infection.

Memory CD8 T cells - initiate the recall response to secondary infection; thus, very important for vaccine development.

CD4 T cells - augment CD8 and B-cell response to influenza infection; also, have their own antiviral effects that are independent of CD8 and B-cell activity.

Regulatory T cells (Treg)- highly suppressive to CD4 and CD8 effector cells; usually required for initiating resolution of the immune response after infection.

\subsection{Innate Immune Response}

The innate response is critical for early protection against influenza virus and plays a key role in the induction and regulation of adaptive immune response. Innate immunity provides a vital bridge between first exposure of the naïve host and subsequent elaboration of specific antibody and T-cell responses, which is extremely important for clearing virus. Viral RNA is recognized through a variety of pattern recognition receptors (PRRs), including Toll-like receptor (TLR)3, TLR7, TLR8, retinoic acid-inducible gene I (RIG)-I and nucleotide-binding domain-like receptor protein (NLRP)3. Once viral RNA is recognized by PRRs, pro-inflammatory cytokines are secreted, such as type I interferons (IFNs), which induce an antiviral state in neighboring cells. Cytokine secretion leads to the recruitment of innate immune cells, which instruct the adaptive immune response for specific killing of influenzainfected cells [4].

Neutrophils are the first wave of recruited immune cells that respond to influenza virus infection; they provide critical protection by acting to limit viral 
replication [5, 6]. Some studies have suggested that neutrophils are mediators of immunopathology and mortality due to their excessive inflammatory response [7, 8]. However, there is also evidence supporting the protective role neutrophils perform in influenza virus infection [4]. In particular, neutrophil-depleted mice had reduced virus elimination from the infected site over time [9]. Additionally, neutrophils have also been shown to be vital in sustaining effective $\mathrm{CD} 8^{+} \mathrm{T}$-cell responses in the respiratory tract of influenza virus-infected mice, which is important for virus clearance [10].

Resident alveolar macrophages in lungs are involved in the first line of defense. In addition to phagocytosis of virions and virus-infected cells, alveolar macrophages contribute to recognition of the virus to present to the adaptive arm of the immune system [10]. Following recognition, alveolar macrophages produce an array of pro-inflammatory cytokines, including tumor necrosis factor (TNF)- $\alpha$, interleukin (IL)-1, IL-6 and IFN- $\alpha / \beta$, which further limit viral replication. In an animal study, mice depleted of alveolar macrophages had exacerbated disease severity, increased virus replication and a higher mortality when infected with influenza virus [11].

Monocytes are one of the predominant cell types upregulated during influenza virus infection and consequently circulate in high numbers. During inflammation, the bone marrow receives signals from pro-inflammatory cytokines and TLR ligands to increase monocyte production. Respiratory epithelial cells secrete monocyte chemoattractant protein (MCP)-1, which facilitates monocyte trafficking into the lungs [12]. Excessive numbers of monocytes drive immune pathology in severe influenza; however, by reducing recruitment of inflammatory monocytes to the lung, there is a decreased $\mathrm{CD}^{+} \mathrm{T}$-cell response leading to compromised viral clearance [13]. Recruited monocytes also respond to influenza virus infection by differentiating into monocyte-derived macrophages and monocyte-derived dendritic cells [14].

Natural killer (NK) cells are granular lymphocytes that contribute to the control of viral replication and production of cytokines that modulate the immune response to influenza virus [15]. The contribution of NK cells to the antiviral immune response has been extensively studied in mouse models of viral infections, demonstrating that NK cells not only contain viral replication by killing infected cells during earlier stages of infection, but also play a critical role during the development of adaptive immunity [16]. Defects in NK cell activity result in delayed viral clearance and increased morbidity and mortality [15].

Dendritic cells are a critical component in early detection of viral infection and transfer of viral antigens to draining lymph nodes, bridging innate and adaptive immunity. The major function of dendritic cells during the antiviral response is to process and present viral antigenic peptides to antigen-specific $\mathrm{CD} 8^{+}$and $\mathrm{CD} 4^{+} \mathrm{T}$ cells to establish an adaptive immune response [17]. Dendritic cells mainly obtain viral proteins via ingestion of virus-infected cells, as few viruses directly infect dendritic cells. Following ingestion, viral particles are mainly presented on MHC class I molecules through a process known as cross-presentation. Cross-presentation is important as it enables dendritic cells to activate $\mathrm{CD} 8^{+} \mathrm{T}$ cells, which are required for viral clearance [18]. 


\subsection{Adaptive Immune Response}

The cellular and humoral arms of adaptive immunity are both activated in response to influenza virus infection. The humoral arm of the adaptive immune response involves B cells, which secrete antibodies to effectively prevent infection by neutralizing the virus [19]. B-cell responses to influenza infection are largely T-dependent, where maximal antiviral IgG responses rely on $\mathrm{CD}^{+} \mathrm{T}$ cells and their interaction with B cells [20].

The cellular arm of the adaptive immune response involves $\mathrm{T}$ cells, which are critical for viral clearance. Following dendritic cell presentation of viral antigens in the draining lymph nodes, naïve virus-specific $\mathrm{T}$ cells undergo a process of activation, proliferation and differentiation to become effector $\mathrm{T}$ cells that can migrate to the site of infection and mediate antiviral immune responses [21]. Infection with influenza virus induces differentiation of effector $\mathrm{CD}^{+}$and $\mathrm{CD} 8^{+} \mathrm{T}$ cells, however the contribution of $\mathrm{CD}^{+} \mathrm{T}$ cells to virus clearance is minor, compared to $\mathrm{CD} 8^{+} \mathrm{T}$ cells. In influenza infection, the primary role of $\mathrm{CD}^{+} \mathrm{T}$ cells involves supporting the activation and differentiation of $\mathrm{B}$ cells, leading to antibody production, and memory responses. Effector $\mathrm{CD}^{+} \mathrm{T}$ cells are major players in viral clearance and are essential for elimination of infected cells [22]. CD8 ${ }^{+}$effector $\mathrm{T}$ cells mediate viral clearance through three distinct mechanisms. First, $\mathrm{CD} 8^{+} \mathrm{T}$ cells can induce lysis of infected cells through exocytosis of perforin and granzyme containing granules. Second, $\mathrm{CD}^{+} \mathrm{T}$ cells can trigger TNF receptor (TNFR) family-dependent apoptosis in infected or stressed cells through either Fas ligand or TNF-related apoptosis-inducing ligand (TRAIL). Third, when encountered with virus-infected cells, $\mathrm{CD}^{+} \mathrm{T}$ cells can produce pro-inflammatory mediators, such as IFN- $\gamma$, which is important for viral clearance [21].

\subsection{Which Host Factor Is Most Directly Linked to Influenza Fatality?}

The preceding sections demonstrate that a myriad of host factors is involved in mounting an immune response to influenza virus. A key question is, among these host factors identified, which host factor is primarily responsible for influencing disease progression? Several studies have addressed this question. A study by Brandes et al. evaluated all the common host factors implicated in influenza pathogenesis [7]. From this evaluation, the authors discovered that a neutrophil-driven response was the most dominant host factor in disease progression. Notably, the authors identified a neutrophil-driven inflammatory loop that could directly amplify tissue damage and exacerbate inflammation. Thus, the authors concluded that excessive neutrophil activation was the main host factor associated with poor outcome in influenza infection.

Another recent study provides further insight into the mechanism underpinning the neutrophil-driven response in severe influenza. In their study, Narasaraju et al. [8] examined neutrophil influx into the infected lung after influenza infection. Their 
findings confirmed that neutrophils were primarily responsible for causing immunopathology, lung inflammation and subsequently the development of acute respiratory distress syndrome (ARDS). Importantly, the authors further discovered that neutrophil extracellular trap (NET) formation correlated with tissue damage. By examining lung histopathology and NET activity, they found that NETs directly caused tissue damage to alveoli, epithelium and the vascular structure in the infected lung. Thus, this study provides strong etiological evidence that aberrant or excessive neutrophil response was the dominant causative factor in severe influenza.

An important caveat of the above studies is that they were mostly performed in animal models. Thus, it was uncertain whether the mechanistic findings obtained from these studies could be applied to infected humans. Two recent human studies have been performed to address this issue. A study by Dunning et al. examined the host response profile in 131 influenza infected humans (with either H1N1 or H3N2 influenza virus). The patient cohort include those with mild infection $(n=47)$, moderate infection $(n=34)$ and severe infection $(n=28)$ [23]. The findings of this study confirmed previous animal studies; they showed that neutrophil response was the primary host factor in determining the course of illness. Indeed, an excessive neutrophil response was consistently found in most patients with severe disease. Another study by Guan et al. investigated the host response profile of severe influenza infection, using a small case-series of patients with severe H7N9 infection [24]. This study also identified neutrophil response as the most critical factor in severe disease. Collectively, these human studies confirm findings from previous animal studies and pinpoint an aberrant neutrophil response as the main cause of disease progression in influenza infection.

\subsection{How Does Influenza Virus Interact with Host Factors?}

Influenza virus likely interacts with systemic host factors (e.g., circulating neutrophils) during the course of infection. Unravelling this interaction is key to understanding influenza pathogenesis and, in particular, to exploring the mechanism by which influenza infection evolves from lung inflammation to multiorgan failure. Most influenza infection begins in airway and the infection spreads locally to other parts of the lung. The mechanism by which the virus interacts with local immune cells is well studied and much of the molecular/cellular details have already been elucidated. By contrast, the mechanism by which influenza virus interacts with host factors outside the lung, such as circulating immune cells, remains relatively unknown. This is largely due to the established dogma that influenza virus does not circulate outside the lung and, by extension, influenza virus is not expected to interact with circulating immune cells (e.g., neutrophils). This 'lung-centric' paradigm has recently been challenged by new evidence. Several studies showed that influenza virus can indeed be carried by immune cells from lung to non-lung tissues [25-27]. These 'virus carrying' immune cells include professional antigen presenting cells such as monocytes, macrophage and dendritic cells [28-30]. These cells pick up influenza virus RNA during their encountering and processing of virus infected cells 


\section{Inside infected alveoli}
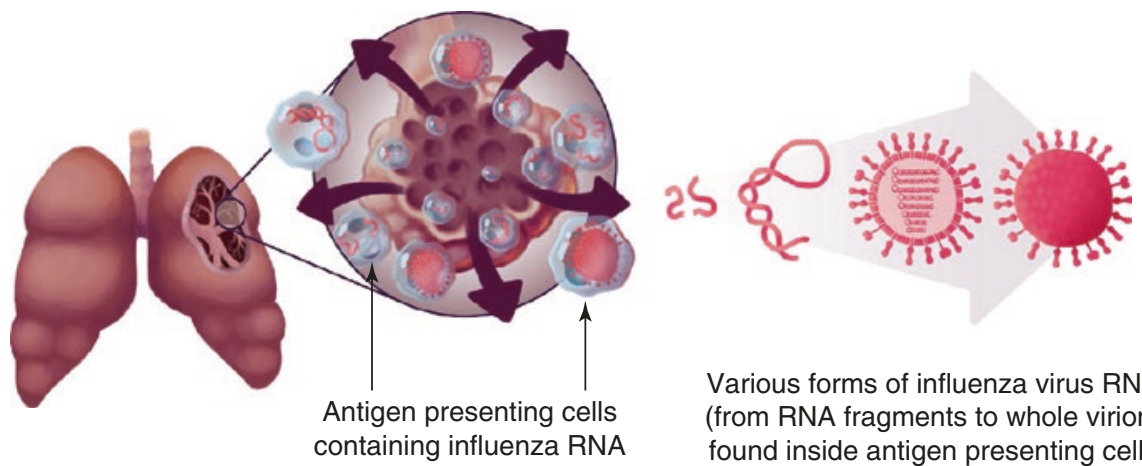

Various forms of influenza virus RNA (from RNA fragments to whole virion) found inside antigen presenting cells

Fig. 35.1 Dissemination of influenza virus from infected lung tissue. Left side: antigen presenting cells carry influenza virus RNA; these 'carrier cells' disseminate influenza RNA from within infected airway/alveoli to outside the lung. Right side: the influenza RNA is carried inside antigen presenting cells in various forms, including (from left to right) (1) a short influenza RNA segment, (2) an intact full influenza RNA segment, (3) a non-replicating whole influenza virus, or (4) a whole influenza virus that is actively replicating and infecting other host cells

(e.g., epithelium) in the early phase of infection (Fig. 35.1). After processing of dead/ infected cells, these cells carry influenza virus RNA within them, migrate towards local draining lymph nodes and subsequently into the systemic circulation.

The influenza virus RNA carried inside these 'carrier' immune cells consists mostly of fragments of the virus RNA (which cannot infect other cells) and a few replicating whole RNA virions (which can infect other cells). Collectively, these RNA molecules are known as the 'influenza virus transcriptome' (Fig. 35.1). Steuerman et al. recently successfully measured the influenza virus transcriptome in infected lung tissues (e.g., epithelium and endothelium) and circulating immune cells (e.g., monocytes, T cells, B cells and NK cells) [31]. Their findings provide strong support to the notion that the influenza virus transcriptome is transported by 'carrier' immune cells (e.g., macrophages or dendritic cells) within the systematic circulation, thereby enabling the dissemination of influenza virus transcriptome loads to distant organs $[28,32]$.

\subsection{Influenza Virus Transcriptome Triggers a Systemic Inflammatory Response}

The influenza virus transcriptome can be cross-presented by 'carrier' immune cells to circulating neutrophils (Fig. 35.2). Neutrophils are recruited to the infected tissue as part of the host response to influenza virus. These cells traffic from the systemic circulation towards the lung, as guided by released of chemoattractants from infected tissue. During this migration, neutrophils encounter 'carrier' immune cells in the systemic circulation, in transit towards the lung or after they have arrived within the infected 
Fig.35.2 Neutrophil extracellular traps (NETs) released by neutrophils upon encountering antigen presenting cells

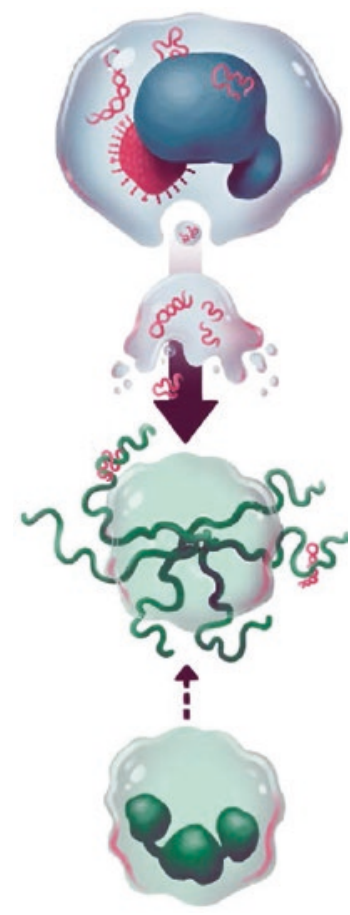

Antigen presenting cells containing processed fragments of influenza RNA or whole virions

Presentation of viral RNA to neutrophils

Neutrophils release histones and fragments of DNA (NETs)

More neutrophils migrate towards site of infection

lung. This encounter triggers a release of a massive amount of NETs (Fig. 35.2). NETs consist of a cellular 'debris' of nuclear contents (histones and DNA fibers) externalized by activated neutrophils in response to bacterial or viral infection.

Although NET formation is an evolutionarily conserved response to microbes, excessive NET release in the infected lung leads to endothelial damage, thrombosis, worsening inflammation. In a recent study, Narasaraju et al. showed that the excessive release of NET in infected lung exacerbated lung inflammation and directly caused the development of acute lung injury [8].

\subsection{From Acute Lung Injury to Multiorgan Failure}

It is clear, from the review above, that lung injury in influenza infection is partly a direct result of the cytopathic effects on host cells (e.g., epithelium or alveoli) by influenza virus and partly an indirect result of a dysregulated host response associated with NETs. Both direct and indirect processes may extend beyond the infected lung, since 'carrier' immune cells can transport influenza virus transcriptome to distant organs where a further encounter with recruited neutrophils may trigger local release of NETs into the affected organs. Indeed, in animal models, influenza virus transcriptome can be detected in major organs in the infected host (heart, spleen, kidney, brain, liver) [32]. Furthermore, a recent study showed that neutrophils can be infected by influenza virus [33]. Once infected, these neutrophils become 'carrier' immune cells themselves. They 


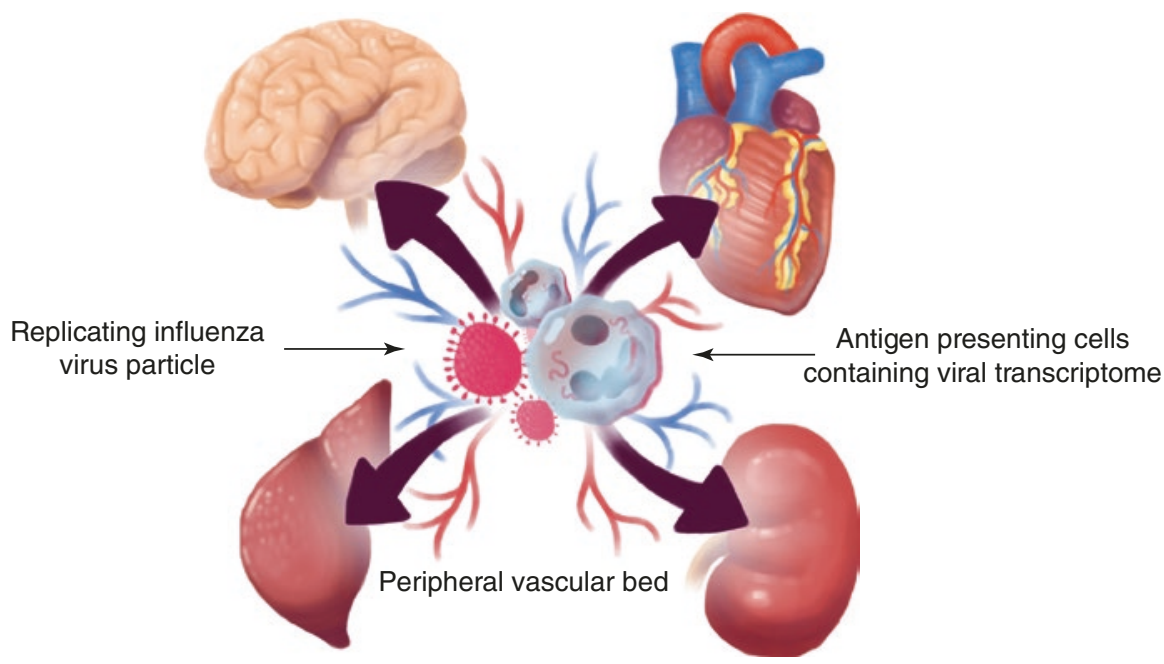

Fig. 35.3 A new multisystem view of influenza pathogenesis

then go on to spread neutrophil-induced inflammation (e.g., NETs, myeloperoxidase, reactive oxygen species) elsewhere or allow actively replicating virions to 'seed' the target organs. Collectively, both direct and indirect tissue injury mechanisms extend an initially lung-centered pathology to other major organs with the ensuing development of organ dysfunction (e.g., myocarditis, acute kidney injury). In light of this, the traditional 'lung-centric' view of influenza pathogenesis requires revision to incorporate a multisystem view of organ dysfunction influenza pathogenesis (Fig. 35.3).

\subsection{Conclusion}

Severe influenza infection remains an important clinical challenge to critical care physicians. The potentially high morbidity and mortality of this condition has remained unchanged over the last few decades, due mainly to a lack of effective new therapies to treat such patients. In recent years, we have gained a much better understanding of the disease mechanism. This improved understanding points to the pivotal roles played by immune dysregulation in causing severe disease. Agents that modulate the host response therefore hold particularly great promise in correcting the dysregulated immune response and may help reduce morbidity/mortality.

\section{References}

1. Thompson WW, Shay DK, Weintraub E, et al. Mortality associated with influenza and respiratory syncytial virus in the United States. JAMA. 2003;289:179-86.

2. Simonsen L. The global impact of influenza on morbidity and mortality. Vaccine. 1999;17(Suppl 1):S3-10. 
3. Taubenberger JK, Morens DM. The pathology of influenza virus infections. Annu Rev Pathol Mech Dis. 2008;3:499-522.

4. Tripathi S, White MR, Hartshorn KL. The amazing innate immune response to influenza A virus infection. Innate Immun. 2015;21:73-98.

5. Camp JV, Jonsson CB. A role for neutrophils in viral respiratory disease. Front Immunol. 2017;8:11.

6. Iwasaki A, Pillai PS. Innate immunity to influenza virus infection. Nature. 2014;14:315-28.

7. Brandes M, Klauschen F, Kuchen S, Germain RN. A systems analysis identifies a feedforward inflammatory circuit leading to lethal influenza infection. Cell. 2013;154:197-212.

8. Narasaraju T, Yang E, Samy RP, et al. Excessive neutrophils and neutrophil extracellular traps contribute to acute lung injury of influenza pneumonitis. Am J Pathol. 2011;179: 199-210.

9. Fujisawa H. Inhibitory role of neutrophils on influenza virus multiplication in the lungs of mice. Microbiol Immunol. 2001;45:679-88.

10. Tate MD, Brooks AG, Reading PC, Mintern JD. Neutrophils sustain effective CD8(+) T-cell responses in the respiratory tract following influenza infection. Immunol Cell Biol. 2012;90:197-205.

11. Kim HM, Lee YW, Lee KJ, et al. Alveolar macrophages are indispensable for controlling influenza viruses in lungs of pigs. J Virol. 2008;82:4265-74.

12. Herold S, von Wulffen W, Steinmueller M, et al. Alveolar epithelial cells direct monocyte transepithelial migration upon influenza virus infection: impact of chemokines and adhesion molecules. J Immunol. 2006;177:1817-24.

13. Cole SL, Ho LP. Contribution of innate immune cells to pathogenesis of severe influenza virus infection. Clin Sci. 2017;131:269-83.

14. Auffray C, Sieweke MH, Geissmann F. Blood monocytes: development, heterogeneity, and relationship with dendritic cells. Annu Rev Immunol. 2009;27:669-92.

15. Jost $S$, Altfeld M. Control of human viral infections by natural killer cells. Annu Rev Immunol. 2013;31:163-94.

16. Schultz-Cherry S. Role of NK cells in influenza infection. Curr Top Microbiol Immunol. 2015;386:109-20.

17. Grayson MH, Holtzman MJ. Emerging role of dendritic cells in respiratory viral infection. $\mathrm{J}$ Mol Med. 2007;85:1057-68.

18. Belz GT, Wilson NS, Kupresanin F, Mount AM, Smith CM. Shaping naive and memory CD8+ $\mathrm{T}$ cell responses in pathogen infections through antigen presentation. Adv Exp Med Biol. 2007;590:31-42.

19. Sealy R, Surman S, Hurwitz JL, Coleclough C. Antibody response to influenza infection of mice: different patterns for glycoprotein and nucleocapsid antigens. Immunology. 2003;108:431-9.

20. Sangster MY, Riberdy JM, Gonzalez M, Topham DJ, Baumgarth N, Doherty PC. An early CD4 +T cell-dependent immunoglobulin A response to influenza infection in the absence of key cognate T-B interactions. J Exp Med. 2003;198:1011-21.

21. Braciale TJ, Sun J, Kim TS. Regulating the adaptive immune response to respiratory virus infection. Nat Rev Immunol. 2012;12:295-305.

22. Sridhar S, Begom S, Bermingham A, et al. Cellular immune correlates of protection against symptomatic pandemic influenza. Nat Med. 2013;19:1305-12.

23. Dunning J, Blankley S, Hoang LT, et al. Progression of whole-blood transcriptional signatures from interferon-induced to neutrophil-associated patterns in severe influenza. Nat Immunol. 2018;19:625-35.

24. Guan W, Yang Z, Wu NC, et al. Clinical correlations of transcriptional profile in patients infected with avian influenza H7N9 virus. J Infect Dis. 2018;368:1888-11.

25. Korteweg C, Gu J. Pathology, molecular biology, and pathogenesis of avian influenza A (H5N1) infection in humans. Am J Pathol. 2008;172:1155-69.

26. Likos AM, Kelvin DJ, Cameron CM, et al. Influenza viremia and the potential for blood-borne transmission. Transfusion. 2007;47:1080-8. 
27. Choi SM, Xie H, Campbell AP, et al. Influenza viral RNA detection in blood as a marker to predict disease severity in hematopoietic cell transplant recipients. J Infect Dis. 2012;206:1872-7.

28. Short KR, Brooks AG, Reading PC, Londrigan SL. The fate of influenza A virus after infection of human macrophages and dendritic cells. J Gen Virol. 2012;93:2315-25.

29. Hou W, Gibbs JS, Lu X, et al. Viral infection triggers rapid differentiation of human blood monocytes into dendritic cells. Blood. 2012;119:3128-31.

30. Hoeve MA, Nash AA, Jackson D, Randall RE, Dransfield I. Influenza virus A infection of human monocyte and macrophage subpopulations reveals increased susceptibility associated with cell differentiation. PLoS One. 2012;7:e29443.

31. Steuerman Y, Cohen M, Peshes-Yaloz N, et al. Dissection of influenza infection vivo by singlecell RNA sequencing. Cell Syst. 2018;7:1-13.

32. Fislova T, Gocnik M, Sladkova T, et al. Multiorgan distribution of human influenza A virus strains observed in a mouse model. Arch Virol. 2009;154:409-19.

33. Zhang Z, Huang T, Yu F, et al. Infectious progeny of 2009 A (H1N1) influenza virus replicated in and released from human neutrophils. Sci Rep. 2015;5:1-11. 